\title{
Mutagenic Activity of AID/APOBEC Deaminases in Antiviral Defense and Carcinogenesis
}

\author{
O. N. Shilova ${ }^{a}$, D. L. Tsyba ${ }^{b, c}$, and E. S. Shilov ${ }^{d, *}$ \\ a Shemyakin-Ovchinnikov Institute of Bioorganic Chemistry, Russian Academy of Sciences, Moscow, 117997 Russia \\ ${ }^{b}$ Pavlov First State Medical University, St. Petersburg, 197022 Russia \\ ${ }^{c}$ Sirius University of Science and Technology, Sochi, 354340 Russia \\ ${ }^{d}$ Faculty of Biology, Moscow State University, Moscow, 119234 Russia \\ *e-mail: shilov_evgeny@inbox.ru.
}

Received December 28, 2020; revised April 23, 2021; accepted June 1, 2021

\begin{abstract}
Proteins of the AID/APOBEC family are capable of cytidine deamination in nucleic acids forming uracil. These enzymes are involved in mRNA editing, protection against viruses, the introduction of point mutations into DNA during somatic hypermutation, and antibody isotype switching. Since these deaminases, especially AID, are potent mutagens, their expression, activity, and specificity are regulated by several intracellular mechanisms. In this review, we discuss the mechanisms of impaired expression and activation of AID/APOBEC proteins in human tumors and their role in carcinogenesis and tumor progression. Also, the diagnostic and potential therapeutic value of increased expression of AID/APOBEC in different types of tumors is analyzed. We assume that in the case of solid tumors, increased expression of endogenous deaminases can serve as a marker of response to immunotherapy since multiple point mutations in host DNA could lead to amino acid substitutions in tumor proteins and thereby increase the frequency of neoepitopes.
\end{abstract}

Keywords: AID, APOBEC, mutagenesis, tumor, RNA editing, genomic instability

DOI: $10.1134 /$ S002689332201006X

\section{INTRODUCTION}

AID/APOBEC (Activation Induced Deaminase/Apolipoprotein B mRNA Editing enzyme, Catalytic polypeptide-like) is a family of $\mathrm{Zn}$-dependent cytidine deaminases that convert cytidine of RNA or single-stranded DNA into uridine. Normally, these enzymes are involved in editing cellular mRNA and the antiviral immune response of the cell. They also provide somatic hypermutation in immunoglobulin genes during B-lymphocyte maturation. In human cells, there are 11 proteins belonging to the AID/APOBEC family, including AID, APOBEC1, APOBEC2, APOBEC4, and 7 homologues of APOBEC3, numbered $3 \mathrm{~A}$ to $3 \mathrm{H}$ with exception of $3 \mathrm{E}$, the expression profile, and function of which are tissue-specific. The cytidine deaminase domains of all enzymes of the AID/APOBEC family are characterized by the presence of a conserved $\mathrm{Zn}$-containing ZDD motif with an $\alpha$-helix- $\beta$-sheet- $\alpha$-helix supersecondary structure [1]. The choice of substrate and regulation of catalytic deamination are controlled by the length, composition, and spatial arrangement of the protein domains adjacent to the catalytic site. Nucleic acids recognized by AID/APOBEC deaminases usually have very short consensus sequences. For example, AID recognizes the motif $\mathrm{WRC}^{*} \mathrm{H}$; APOBEC $3 \mathrm{~A}$,
TTC*A; APOBEC3B, ATC*A; APOBEC3F, TTC*W; APOBEC $3 \mathrm{G}, \mathrm{YCC}^{*} \mathrm{H}$; and APOBEC $3 \mathrm{H}, \mathrm{TC}^{*} \mathrm{~A}$ (asterisks indicate cytosine deamination) [2]. The range of possible targets with such sequences is extremely wide, therefore, dysregulation of expression and mutations in APOBEC deaminase genes cause a large number of changes in various DNA and mRNA sequences and lead to the development of a number of immunological disorders (hyper-IgM syndrome), as well as malignant neoplasms (B-cell lymphomas, hepatocellular carcinoma, and other tumors). The ability of enzymes to modify nucleic acids is used by the cell to change its phenotype at the nuclear genome or mRNA editing level. However, the same enzymes, with insufficient selectivity or inaccurate regulation, can generate oncogenic mutations and contribute to malignant cell transformation. This review is devoted to the role of deaminases of the AID/APOBEC family in carcinogenesis and tumor progression.

\section{THE ROLE OF AID/APOBEC DEAMINASES IN EDITING CELLULAR RNA AND DNA}

The first member of the family described was the APOBEC1 enzyme, which, with the participation of cofactors, edits apolipoprotein B (apoB) mRNA in 
epithelial cells of the small intestine, which is reflected in the family name. As a result of the conversion of cytidine to uridine, a stop codon is formed in the mRNA sequence, and the final protein product is 2 times shorter than the full-length one. Both forms of apoB ensure the absorption, transport, and consumption of triglycerides and cholesterol by tissues, although they are included in different structures. The truncated variant is present only in chylomicrons that transport lipids from the intestine to the liver [3] since APOBEC1 is expressed in the small intestine, but not in hepatocytes. In turn, the full-size protein serves as the structural basis for the lipoprotein particle and remains in it during its entire existence [4,5]. APOBEC2 is involved in the differentiation of skeletal and cardiac muscles [6-8] and is involved in the regulation of transforming growth factor- $\beta$ (TGF- $\beta$ ) signaling, which determines lateral asymmetry during embryonic development of vertebrates, although knockout is not lethal $[9,10]$. The importance of APOBEC2 for the normal functioning of mitochondria in muscles has been reported, however, the molecular substrate of this enzyme has not yet been identified and the detailed mechanism of its operation is unknown [11]. APOBEC3 deaminases are expressed in cells involved in the antiviral immune response and edit single-stranded viral nucleic acids by introducing mutations. The APOBEC4 protein is predominantly expressed in the testes, but its function is still unknown [12].

AID deaminase alters the phenotype of a cell by introducing mutations into genomic DNA. This enzyme is involved in somatic hypermutation accompanying VDJ recombination at the immunoglobulin loci of B lymphocytes, in antibody isotype switching, and in B cell affinity maturation [13]. Deoxyuridine in DNA is formed by the AID enzyme mainly during the G1 phase of the cell cycle [14, 15], during which it is also recognized by the repair systems. During the $\mathrm{S}$ phase, most of the changes in the DNA sequence are preserved as a transition and, less often, as a transversion; the likelihood of mutation in a locus affected by deaminase increases from $10^{-9}$ to $10^{-3}$ per nucleotide per division. When deamination sites are close, the work of the excision repair system can lead to doublestranded DNA breaks. Then, they are repaired, which leads to a rearrangement of the locus and a change in the isotype of the encoded antibody [16]. The activation of AID in the B cell is caused by events such as the activation of the B-cell receptor against the background of additional signals from the co-stimulatory molecule CD40, or TLR, or cytokines influencing the choice of the antibody class: interleukin-4 (IL-4), TGF- $\beta$, or interferon- $\gamma($ IFN- $\gamma$ ) [17]. The main transcription factors that trigger AID expression are $\mathrm{NF}-\kappa \mathrm{B}$ and HoxC4 [17]. Regulation of the activity of this deaminase is shown schematically in Fig. 1.

\section{MECHANISMS OF REGULATION OF AID/APOBEC ACTIVITY}

Many enzymes are regulated not only by the expression of the genes encoding them but also at the post-translational level. This also applies to the AID/APOBEC proteins. Intracellular localization is one of the important factors regulating the activity of cytidine deaminases. During interphase, the APOBEC3A, APOBEC 3C, and APOBEC $3 \mathrm{H}$ proteins are distributed more or less evenly in the cell, possibly due to their small size $(\sim 25 \mathrm{kDa})$, which allows nonspecific penetration through nuclear pores [18]. APOBEC3 proteins, which have two deaminase domains and a molecular weight of about $50 \mathrm{kDa}$, can be both nuclear (APOBEC3B) and cytoplasmic (APOBEC3D, APOBEC 3F, and APOBEC $3 \mathrm{G}$ ) during interphase [18]. AID, despite its small size, is located mainly in the cytoplasm, since it is removed from the nucleus by the CRM1 exportin, which recognizes the nuclear export signal (NES) within the C-terminal motif, and requires active nuclear localization signal-mediated (NLS) import to enter the nucleus [19, 20]. After the transfer of AID into the nucleus, the protein remains there for a short time, about $30 \mathrm{~min}$; the rest of the time, deaminase is located mainly in the cytoplasm [21]. An important signal for changing the localization of AID is its phosphorylation by protein kinase A: the chromatin-associated form of AID is predominantly phosphorylated at the Ser38 residue [22-24]. APOBEC1 can also move to the nucleus, but in the absence of $\mathrm{ACF}$, a partner protein, it is mainly located in the cytoplasm [25, 26]. Interestingly, DNA damage can cause the transfer of APOBEC $3 \mathrm{G}$ from the cytoplasm to the nucleus [27], as well as the transfer of AID in the same direction [28]. The cytoplasmic localization of most APOBEC3 appears to be associated with the editing of cytoplasmic nucleic acids, including viral nucleic acids. Another cell compartment containing nucleic acids is mitochondria; however, the data on whether cytidine deaminases are capable of introducing mutations into mitochondrial DNA are contradictory. In nasopharyngeal cancer induced by the Epstein-Barr virus, induction of APOBEC $3 B$ and APOBEC3F, which correlated with the frequency of $\mathrm{G} \rightarrow \mathrm{A}$ transitions in mitochondrial DNA, was found [29]. However, the analysis of mitochondrial DNA from B-cell lymphoma lines with high levels of AID expression reveals almost no mutations caused by cytidine deaminases [30]. It is assumed that mitochondrial DNA is deaminated by cytidine deaminases exclusively in the cytoplasm [31].

The mechanisms providing AID selectivity for immunoglobulin genes are not fully understood. The transcription factors ETS1 and PAX5 have been shown to play a key role in the recruitment of AID to the immunoglobulin heavy chain sequences in mice; the proteins E2A and IRF4 are also involved in the formation of the complex [32]. In mice, ETS1 is 


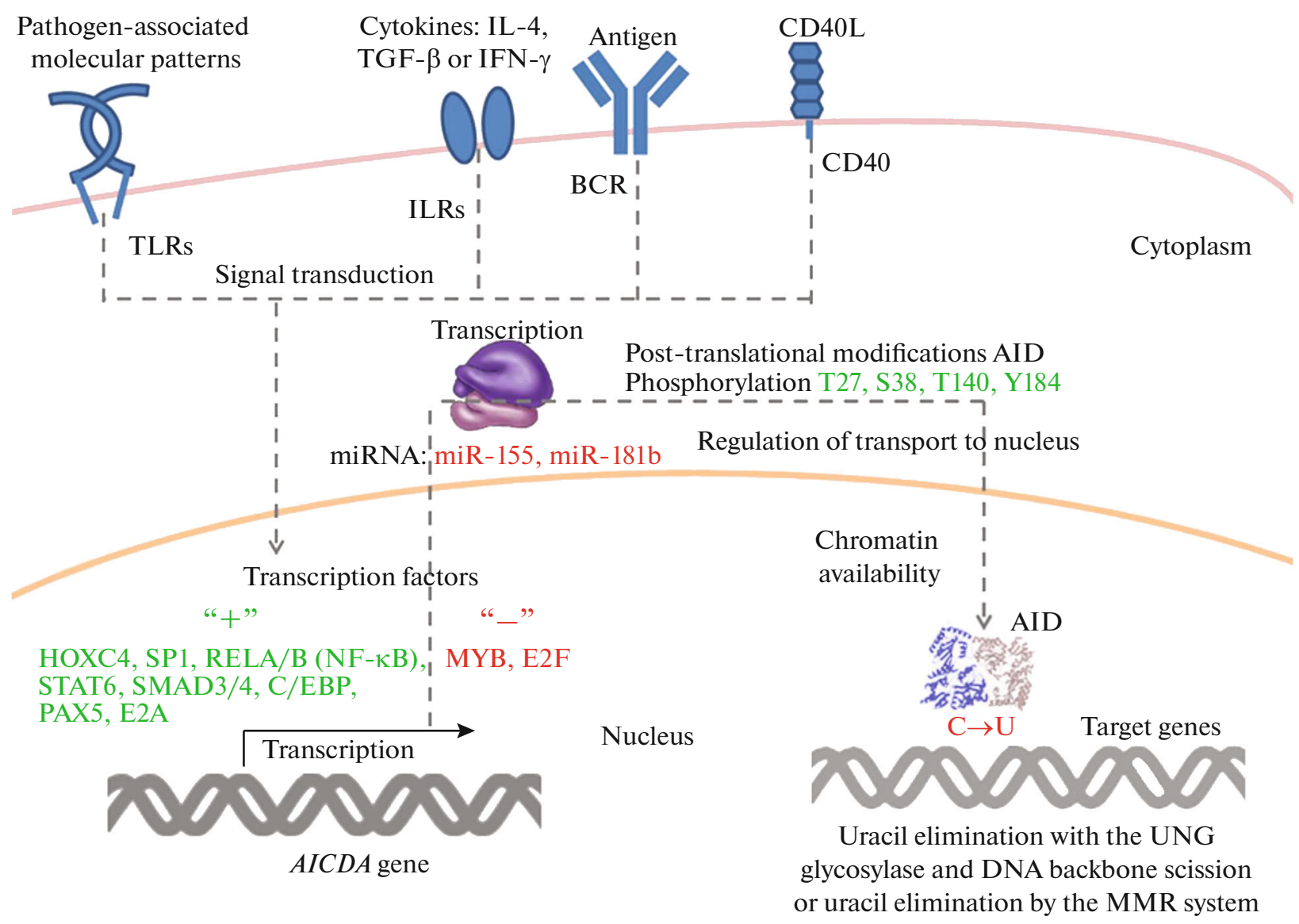

Fig. 1. Diagram of regulation of the mutagenic activity of cytidine deaminases using AID as an example. TLRs, Toll-like receptors; ILRs, interleukin receptors, BCR, B-cell receptor; UNG, uridine nucleotide glycosylase; MMR, miRNA-microRNA mismatch repair.

expressed in many tissues during embryonic and early postnatal development; however, in adult mice and humans, the expression of this protein is observed mainly in the organs of the immune system: thymus, spleen, and lymph nodes. Interestingly, the level of ETS1 is relatively high during the "resting" stages of B-cell development (pre-B cells, pro-B cells, $\operatorname{IgM}^{+}$B cells of the red bone marrow, naive $\mathrm{B}$ lymphocytes, and memory B cells), but decreases upon activation of $B$ cells. B cells of germinal centers and plasma cells are characterized by low expression or complete absence of ETS1 [33]. ETS1 itself upregulates the expression of the B-cell-specific transcription factor PAX5, which is also involved in maintaining the precise localization of AID. A decrease in the expression of ETS1 is a necessary condition for further differentiation of B cells, since ETS1 inhibits the BLIMP-1 factor, which ensures the development of plasma cells [34]; in turn, BLIMP-1 decreases the expression of PAX5 [35]. Thus, the expression of proteins that determine the selectivity of deaminase in relation to immunoglobulin genes decreases simultaneously with the decrease in the expression of AID in cells of germinal centers. It can be assumed that in the absence of proteins that limit the activity of AID, the number of randomly edited regions of the genome will increase. However, it is not known how selective deaminases are towards target genes in the case of their pathological activity. Most likely, the choice of targets is largely random and is primarily determined by the spatial availability of DNA for deaminase within the nucleus. Thus, using sequencing, Meng et al. [36] found that sequences edited by AID in mouse and human B lymphocytes are associated with regions of active transcription, namely, regions transcribed in both directions comprising super-enhancers. Qian et al. [37], based on the results on chromatin immunoprecipitation, also showed that DNA regions subjected to random AID editing are associated with active regions of superenhancers, which, in turn, depend on the cell type.

\section{ANTIVIRAL FUNCTION OF THE APOBEC3 DEAMINASES}

For most members of the AID/APOBEC family, either cell mRNA editing has not been demonstrated, as is the case with APOBEC2 and APOBEC4, or preferential editing of viral nucleic acids has been shown, 
as is the case for the APOBEC3 proteins. The antiviral activity of deaminases has been described in most detail for APOBEC3G, namely for the interaction of APOBEC3G with the HIV genome. The APOBEC3G protein enters viral capsids being packed, binds to the proviral single-stranded minus strand of DNA, and deaminates cytidine mainly in the 5'-Cㅡ- 3 ' sequences [4]. Subsequently, RNA-dependent DNA polymerase recognizes uracil as thymine, which leads to the substitution of guanine for adenine during the synthesis of the plus-strand of DNA in a new infected cell. The associated hypermutation leads to the formation of premature stop codons and the accumulation of other mutations that prevent the formation of new viral particles [38]. Thus, APOBEC3G influences the multiplication of the virus in the next cycle of replication after entering the cell. Also, deamination of cytosine can reduce the amount of viral DNA in the infected cell due to the recognition and destruction of uracilcontaining DNA by the repair systems [39]. The related proteins $\mathrm{APOBEC} 3 \mathrm{~F}, \mathrm{APOBEC} 3 \mathrm{D}$, and APOBEC $3 \mathrm{H}$ are also involved in counteracting retroviruses by creating mutations at other sites in the viral DNA (5'-TC-3') [4]. For proteins of the APOBEC3 family, inhibition of reverse transcription independent of deaminase activity has been described as an alternative mechanism for limiting the replication of lentiviruses [40]. However, this hypothesis has not been confirmed in other works, since the APOBEC $3 \mathrm{G}$ protein lacking a catalytic domain did not suppress HIV infection [41, 42].

During the co-evolution of viruses and hosts, some viruses have acquired resistance to cellular defense systems. For example, viruses can inactivate deaminases, preventing them from packaging into the virion, and successfully continue the infection. Thus, the Cterminal sequence of the human T-lymphotropic virus nucleocapsid protein contains two short sequences that normally prevent APOBEC3G molecules from entering new virions and thereby reduce the virus's sensitivity to the action of deaminases [43]. Foamy viruses (Spumaretrovirinae) rely on a special protein Bet, which interacts with APOBEC $3 \mathrm{G}$ and $\mathrm{APOBEC} 3 \mathrm{~F}$ and prevents packaging of these proteins into the virion, although it does not reduce their content in the cytoplasm of the infected cell [44]. Finally, the most radical solution to the problem of deaminases has been developed as a result of the evolution of HIV-1. The viral protein Vif can bind to proteins APOBEC3C, D, F, G, and $\mathrm{H}$ and attract specific E3-ubiquitin ligase, which leads to degradation of deaminases in the proteasome [4]. However, Vif is unable to destroy the entire APOBEC3 pool in the infected cell. This is indicated by multiple $G \rightarrow A$ substitutions at specific sites of proviruses in the genomes of cells obtained from patients with acute and chronic HIV infection, as well as during vertical transmission of HIV to newborns [45].

Analysis of single amino acid substitutions made it possible to identify the key human APOBEC 3G regions required for the interaction of HIV with 1Vif: the Asp128 residue is required for binding; Pro129 and Asp130 are also important [46]. Vif proteins of other mammalian lentiviruses bind in the same region of APOBEC3G, although the key residues may differ [47]. For packaging into virions, amino acids 124-127 of APOBEC3G, which are immediately adjacent to the Vif recognition site, are important [46]. Genes of mutant APOBEC $3 \mathrm{G}$ variants are used in the development of gene therapy for HIV infection: the sequence of the Vif-resistant APOBEC3G protein is introduced into $T$ cells or stem cells of red bone marrow intended for transplantation in order to enhance the natural mechanism of cell defense against the retrovirus [48]. Oddly enough, lentiviral vectors, being among the most efficient in terms of transduction and capable of integrating the resistant Vif gene into the genome of the target cell, are damaged by APOBEC $3 \mathrm{G}$ in the producer cells, which requires the use of an adenoassociated virus [49] or the use of self-activating lentivirus systems [50].

Despite the protective role of wild-type APOBEC3 deaminases, mutagenesis caused by them can serve as a source of mutations beneficial for the virus. HIV strains that carry some Vif mutations that reduce its activity acquire resistance to antiretroviral therapy more often due to more frequent $\mathrm{G} \rightarrow \mathrm{A}$ transitions, including those in drug target genes [51]. Apparently, in this case, deaminases cannot completely suppress viral replication, but ensure a high level of mutagenesis, which provides material for the selection of more aggressive strains. In addition, the residual activity of APOBEC 3 can affect the sites that influence the binding of antigenic peptides of viral proteins to molecules of the major histocompatibility complex [52]. In the course of further study of proteins of the APOBEC3 family, data have been accumulated on the antiviral action of these enzymes against retroviruses, including human T-lymphotropic virus [43], retrotransposons [53], simian immunodeficiency virus [54], equine infectious anemia virus [55], murine leukemia virus [56], spumaretrovirus [57, 58], and adeno-associated virus [53]. The study of the interaction of APOBEC3 proteins with DNA, as expected, led to the establishment of the role of these proteins in the containment of infections caused by DNA-containing viruses, such as hepatitis B virus [59, 60] and parvoviruses [53]. In addition to deamination of single-stranded DNA, the ability of APOBEC3 deaminases to edit HIV-1 RNA was found [61], which suggests such activity against other RNA-containing viruses that do not have a reverse transcription stage in the replication cycle. In addition, RNA editing mediated by APOBEC 3 deaminases was found for M1-type activated monocytes and macrophages [62], as well as breast cancer cells. In the latter case, the editing intensity correlated with the level of activation of proinflammatory protein genes and the number of immune cells migrating to the tumor lesion [63]. 
Recently, APOBEC1 has been shown to be able to edit chromosomal DNA [64]; therefore, the previous functional division of the enzymes into DNA- and RNA-editing is arbitrary. Indeed, for RNA-containing viruses of mumps, measles, and respiratory syncytial virus, a decrease in the efficiency of infection of cells overexpressing APOBEC3G has been demonstrated [65]. It is possible that other proteins of the APOBEC3 family also play a role in this. Thus, infection of respiratory epithelial cells with the $\mathrm{HCoV}$ NL63 coronavirus led to increased expression of APOBEC $3 \mathrm{~A}, 3 \mathrm{C}, 3 \mathrm{D}$, and $3 \mathrm{~F}$, but not $3 \mathrm{G}$, and the expression of $3 \mathrm{C}, 3 \mathrm{~F}$, and $3 \mathrm{H}$ proteins in LLC-Mk2 cells reduced the viral load by two orders of magnitude; the protective effect was observed only in the case of catalytically active proteins [66]. Nevertheless, most mammals, including rodents, manage just fine with a single copy of APOBEC3, although the presence of seven different paralogs in humans may be associated with the specificity of deaminases for nucleic acids of various viruses.

\section{MECHANISMS OF AID/APOBEC EXPRESSION IMPAIRMENT IN TUMORS}

Deaminases of the AID/APOBEC family are an endogenous source of mutations in a cell; therefore, for a cancer cell, they are suppliers of new mutations, which are then subjected to selection at the level of tumor clones. Analysis of genome-wide tumor sequencing data revealed APOBEC clusters of focal hypermutation in cells of many types of cancer, such as squamous cell lung cancer, bladder cancer, acral melanoma, and some sarcomas, and the presence of these clusters positively correlated with the level of APOBEC3B expression $[67,68]$. The association of AID/APOBEC proteins with the formation of a tumor clone may be direct, through mutations in the deaminase gene leading to an increase in its activity. The role of mutations in the APOBEC3 genes (paralogs C, D, F, G, and H) in the pathogenesis of multiple myeloma has been revealed [69]; mutations in the genes of deaminases can increase their mutagenic potential in tumors. The appearance of single nucleotide polymorphisms and deletions in APOBEC3 genes correlates with an increased risk of developing bladder and breast cancer, respectively [70-72]. However, most often violations of regulatory mechanisms, which normally maintain the expression of deaminases at a level that is safe for the cell, are observed. When considering individual members of the family, one can notice that for almost all of them there are reports of either overexpression or mutations in tumors.

Most obvious is the mutagenic activity of AID deaminase. Normally, it provides rearrangement of three loci $(I G H, I G L$, and $I G K)$ encoding heavy and light chains of antibodies; if this process is disturbed, B-cell lymphomas arise, for example, Burkitt's lymphoma, which appears as a result of an AID-depen- dent fusion of the $I G H$ and $M Y C$ loci. However, nonspecific rearrangements can also occur in other regions of the genome, leading to oncogenic mutations [73, 74]. Constitutive expression of AID in mice leads to the development of T-cell lymphomas and lung tumors [75]. It is not surprising, therefore, that increased expression of AID is often found in malignant lymphocytes, for example, in tumor cells of B-cell lymphocytic leukemia. Increased expression of AID is associated with a poor prognosis for the patient $[76,77]$ and does not necessarily correlate with the presence of rearrangements in the genes of heavy immunoglobulin chains [76]. It is possible that the pattern of mutations arising in a malignant lymphocyte is influenced by the expression of alternative AID splice forms, which is often observed in chronic lymphocytic leukemia [78]. For example, the absence of rearrangements in the heavy chain locus is more common in the case of increased expression of three alternative AID splice forms at once [79]. Expression of AID was observed in some stomach, lung, liver, ovary, and breast tumors [80, 81].

In addition to lymphocytes, proteins of the AID/APOBEC family can normally be expressed in many cell types in response to viral infection. In this case, under the action of proinflammatory cytokines in the cell, the expression and activity of deaminases that suppress viral replication are increased, as already described above. The NF- $\kappa \mathrm{B}$ signaling pathway $[82,83]$, which is also involved in the activation of AID [84, 85], is one of the key intracellular pathways for the regulation of APOBEC 3 expression. For APOBEC3A, APOBEC3F, and $A P O B E C 3 G$, an increase in expression in response to IFN $\alpha$ has also been shown [86-88]. However, the high activity of APOBEC does not always allow the cell to eliminate the virus, and parallel somatic mutagenesis in combination with viral damage can lead to dysregulation of the cell cycle and malignant transformation of the cell. In addition, not only can inflammation increase the activity of antiviral proteins of the APOBEC family, but also an aberrant expression of AID may occur. Increased expression of APOBEC has been described in virus-associated stomach, liver, and cervical tumors [89]. Some viruses can stimulate the expression of APOBEC proteins by themselves. The Epstein-Barr virus encodes the LMP1 protein, which triggers the CD40 signaling pathway regardless of the presence of a ligand, which leads to the activation of NF- $\mathrm{KB}$ and the expression of AID [90, 91]. Infection of cultured cells with hepatitis $\mathrm{C}$ virus also induces the expression of AID and, in addition, the SOS response DNA repair polymerases $\zeta$ and 1 . At the same time, rearrangements in the IGH immunoglobulin locus and accumulation of mutations in the genes encoding BCL-6, p53, $\beta$-catenin, and $\beta$-globin are observed [92]. At least some of these mutations are caused by AID activity; therefore, it can be assumed that this enzyme contributes to the development of neoplasms associated with the hepatitis $\mathrm{C}$ 
virus, including non-Hodgkin B-cell lymphomas and oligoclonal lymphocyte proliferation [93]. However, a viral infection of a cell is far from the only stimulus that triggers the activation of NF- $\mathrm{KB}$. Signaling pathways of this system include receptors for TNF, lipopolysaccharide, and IL-1 (canonical pathway), as well as activation signals for lymphocyte proliferation, such as BAFFR, CD40, RANK, and LT $\beta$ R (noncanonical pathway) [94]. Therefore, it is not surprising that increased expression of proteins of the APOBEC family has been detected in many types of cells in the context of inflammation processes of various etiologies.

An increase in the expression of APOBEC deaminases in response to proinflammatory cytokines is, apparently, a fairly common phenomenon that is observed in different types of cells. In hematopoietic cells ( $\mathrm{T}$ lymphocytes, macrophages, dendritic cells), the expression of most APOBEC 3 proteins (except for APOBEC3B and APOBEC3C) is increased by the action of proinflammatory cytokines: IFN $-\alpha$, IFN $-\gamma$, IL-2, IL-15, TNF $\alpha$ [95]. A similar increase in APOBEC 3G expression in response to the action of IFN- $\alpha$, IFN- $\gamma$, IL- 1 , and TNF $\alpha$ was shown for neuronal and astrocytic cells [96], as well as for epithelial cells $[97,98]$. Bacterial pathogens also cause an increase in the expression of proteins of the APOBEC family through the activation of NF- $\mathrm{KB}$. It is known that Helicobacter pylori strains containing virulence factors cagPAI not only provoke the development of gastritis and stomach ulcers but are also involved in the pathogenesis of gastric cancer and lymphoma originating from mucous-associated lymphoid tissue [99]. One of the mechanisms of $H$. pylori-induced carcinogenesis may be an aberrant expression of AID in gastric epithelial cells, which was found in the presence of pathogenic strains of $H$. pylori leading to the accumulation of mutations in the epithelial cell genome, including the p53 tumor suppressor gene [81]. In this case, two mechanisms contribute to the induction of AID: direct activation of NF- $\kappa \mathrm{B}$ by virulence factors, which the bacterium introduces into the epithelial cell using a type IV secretion system, and triggering of an inflammatory response mediated by cytokines [98].

Chronic inflammation that is not clearly associated with a specific pathogen can also cause aberrant expression of AID/APOBEC, which contributes to malignancy. Bowel inflammation caused by ulcerative colitis or Crohn's disease significantly increases the risk of colorectal cancer, with the likelihood correlating with the duration of the disease [100, 101]. In the absence of infection and signals from the microbiota, proinflammatory cytokines can be produced by the tumor microenvironment, contributing to the accumulation of mutations and the formation of subclones within the tumor. Overexpression of APOBEC is characteristic of head and neck, lungs, cervix, breast, and bladder tumors not associated with pathogens [102]. It is believed that in these types of tumors, deaminases of the APOBEC family do not belong to oncogenes as such, but contribute to tumor progression. For example, in the cells of the intestinal epithelium the Th2 cytokines IL-4 and IL-13 can trigger AID expression, leading to the accumulation of mutations in the p53 tumor suppressor gene [97]. In turn, disruption of p53 function can enhance the aberrant expression of deaminases. The promoter regions of the APOBEC3 genes contain p53 binding sites, which normally leads to a decrease in gene expression. In this regard, mutations of the TP53 gene, or blocking of its protein product, for example, by the E6/E7 proteins of the human papillomavirus (HPV), lead to the removal of its inhibitory effect and an increase in the expression of APOBEC [103]. For a number of tumors, overexpression of APOBEC proteins was revealed only at the late stages of progression, when the tumor deeply invades the surrounding tissues and metastasizes [104]. For example, in primary lung adenocarcinomas, the profile of mutations did not correspond to the activities of APOBEC, while in metastases a significant amount of mutations can be explained by the action of deaminases [105]. An increase in the expression of APOBEC proteins may be due to a decrease in the inhibitory effect of p53 in the case of virus-associated tumors. Thus, some viruses (in particular, HPV) can induce the expression of APOBEC [106] by removing the inhibitory effect of the $\mathrm{p} 53$ protein. This further enhances the expression of APOBEC 3A and APOBEC3B, initially caused by the activation of antiviral immunity, which ultimately leads to the emergence of somatic mutations and the formation of a tumor cell clone, worsening the course of the disease and prognosis [107]. Increased expression of APOBEC3B and the presence of single or cluster mutations in target sequences are revealed in the analysis of bladder, cervical, lung, and breast cancer cells and head and neck tumors [67]. The presence of a large number of genetic rearrangements should lead to the activation of repair systems or cell death by apoptosis; however, in tumor cells, these defense mechanisms are impaired, and increased mutagenesis leads to tumor progression.

Increased expression in tumors has been found even for those members of the AID/APOBEC family whose natural targets remain unknown. For example, although there are no data on the effect of APOBEC1 on carcinogenesis in humans, a correlation was found between the level of APOBEC1 expression and the number of insertions/deletions in the genomes of cancer cells [108]. APOBEC2 is usually considered solely in light of embryonic development of striated muscle tissue, since defects in this enzyme molecule are associated with myopathies. However, there are reports on the relationship of aberrant APOBEC2 expression in hepatocytes with the development of hepatocellular carcinoma against the background of hepatitis B [109]. Infection of hepatocytes with hepatitis B virus is assumed to lead to disruption of the formation of regulatory RNAs and an increase in the expression of promutagenic deaminase. The expression of APOBEC3 proteins in tumor 
cells can be negatively regulated at the posttranscriptional level through binding of APOBEC mRNA to microRNA in the cytoplasm, which prevents the normal synthesis of the protein product [110].

Point mutations in DNA caused by the tandem of cytidine deaminases and repair systems can lead to inactivation of tumor suppressor genes, activation of protooncogenes, accumulation of neutral somatic mutations, and the emergence of cancer neoantigens. In the latter case, high deaminase activity means a more likely patient response to tumor immunotherapy and is considered a favorable prognostic marker [111]. In the model of murine melanoma B16, this was confirmed experimentally: the expression of APOBEC3B led to an increase in the number of tumor neoepitopes and increased the effectiveness of immunotherapy [112]. However, one cannot speak of a positive effect of deaminase-mediated mutagenesis. Determination of mutational profiles of tumors and identification of characteristic sets of mutations ("signatures") allows prediction of the clinical course of the disease. Thus, in the case of multiple myeloma, a high level of APOBECmediated transitions gives an unfavorable prognosis $[113,114]$. A similar observation was made for APOBEC3B in cohorts of patients with adrenal carcinoma selected from the Cancer Genome Atlas database [115] and patients with ovarian cancer [116].

\section{CONCLUSION}

Deaminases of the AID/APOBEC family are used by cells to edit their own nucleic acids in the nucleus or to introduce mutations in viral nucleic acids in the cytoplasm. AID makes changes in the genomic DNA of B lymphocytes, which leads to somatic hypermutation or switching of the antibody isotype. Nuclear localization is also characteristic of APOBEC1, which edits apoB mRNA. APOBEC3 proteins work in the cytoplasm and primarily edit viral nucleic acids, suppressing viral replication. Normally, the activity of these deaminases is regulated at several levels: transcription, translation, intracellular localization, target accessibility, and activation within protein complexes. However, the expression of these genes can vary widely during carcinogenesis. The main mechanisms that link tumor induction or progression to the activity of AID/APOBEC deaminases include the following:

(1) transient activation of deaminases during the immune response to a pathogen that does not replicate directly in these cells (as in the case of stomach cancer and Helicobacter pylori);

(2) chronic activation of APOBEC in cells infected with oncogenic viruses (such as HPV and hepatitis B virus) - in this case, the activity of AID/APOBEC proteins, induced directly by viral infection or cytokines secreted by the environment, can lead to the emergence of additional mutations in genomic DNA;
(3) disruption of normal somatic hypermutation during maturation of B lymphocytes and subsequent constitutive or periodic expression of AID deaminase.

As an additional mechanism for the involvement of AID/APOBEC deaminases in carcinogenesis, the occurrence of phenocopies of oncogene mutations or tumor suppressors can be assumed. Since many AID/APOBEC proteins are characterized by the ability to recognize and modify RNA, it can be assumed that mRNA editing creates phenocopies of mutations in DNA. In this case, there will be no mutations in nuclear DNA and they will not be detected during sequencing; however, the mutant phenotype will be realized at the level of transcript editing and in the functioning of proteins translated from these mRNAs. This mechanism is well known for the ADAR family of deaminases, which convert adenosine to inosine. Overexpression of ADAR and specific RNA editing are characteristic of a wide range of tumors and a large number of target genes, including microRNA genes [117]. The role of RNA editing in tumors using adenosine deaminases is discussed in more detail in the review by Gallo et al. [118]. For the APOBEC1 deaminase, a similar mechanism has been identified in tumors of the nervous tissue and mRNA of the neurofibromatosis tumor suppressor NF1 [119, 120]; for APOBEC3, editing of mRNA of many tumor-associated genes in tumors, including $A T M, B A R D 1$, BRCA1, BRCA2, BRIP1, MDM2, KMT2A, MSH2, PTEN, and TSC2 has been demonstrated [121, 122].

Nevertheless, despite the fact that for many types of tumors an increased expression or modified localization of AID/APOBEC deaminases is shown, in most cases their significance for oncogenesis has not been established. It is also unknown how selective deaminases are to target genes when their normal localization is disturbed. Most likely, the choice of the edited sequence is largely random and is primarily determined by the transcriptional activity of the cell. Thus, deaminases contribute to the accumulation of mutations, and selection for oncogenicity of mutations occurs at the level of survival and proliferation of clones within a tumor.

AID/APOBEC deaminases are components of genetic instability, and therapy aimed at modulating their activity is unlikely to have clinical significance. However, as a prognostic marker that allows one to assess the mutagenic potential of tumor cells and the effectiveness of immunotherapy directed against tumor neoantigens, these enzymes are already in demand $[111,113,116]$.

\section{FUNDING}

This work was supported by the Russian Foundation for Basic Research (project no. 19-34-51014) and by the grant of the President of the Russian Federation no. ST-5585.2018.4 carried out using the infrastructure of NTU "Sirius". 


\section{COMPLIANCE WITH ETHICAL STANDARDS}

Conflict of interests. The authors declare that they have no conflicts of interest.

Statement on the welfare of humans or animals. This article does not contain any studies involving humans or animals performed by any of the authors.

\section{REFERENCES}

1. Betts L., Xiang S., Short S., Wolfenden R., Carter Jr. C. 1994. Cytidine deaminase. The $2.3 \AA$ crystal structure of an enzyme: transition-state analog complex. J. Mol. Biol. 235 (2), 635-656.

https://doi.org/10.1006/jmbi.1994.1018

2. Silvas T.V., Schiffer C.A. 2019. APOBEC3s: DNA-editing human cytidine deaminases. Protein Sci. 28 (9), 1552-1566. https://doi.org/10.1002/pro.3670

3. Nakajima K., Nagamine T., Fujita M., Ai M., Tanaka A., Schaefer E. 2014. Apolipoprotein B-48: A unique marker of chylomicron metabolism. Adv. Clin. Chem. 64, 117-177.

4. Salter J.D., Bennett R.P., Smith H.C. 2016. The APOBEC protein family: united by structure, divergent in function. Trends Biochem. Sci. 41 (7), 578-594.

5. Smith H.C. 2017. RNA binding to APOBEC deaminases: Not simply a substrate for $\mathrm{C}$ to $\mathrm{U}$ editing. $R N A$ Biol. 14 (9), 1153-1165.

https://doi.org/10.1080/15476286.2016.1259783

6. Liao W., Hong S.H., Chan B.H., Rudolph F.B., Clark S.C., Chan L. 1999. APOBEC-2, a cardiacand skeletal muscle-specific member of the cytidine deaminase supergene family. Biochem. Biophys. Res. Commun. 260, 398-404.

7. Etard C., Roostalu U., Strahle U. 2010. Lack of Apobec2-related proteins causes a dystrophic muscle phenotype in zebrafish embryos. J. Cell Biol. 189, 527-539.

8. Sato Y., Probst H.C., Tatsumi R., Ikeuchi Y., Neuberger M.S., Rada C. 2010. Deficiency in APOBEC2 leads to a shift in muscle fiber type, diminished body mass, and myopathy. J. Biol. Chem. 285, 7111-7118.

9. Vonica A., Rosa A., Arduini B.L., Brivanlou A.H. 2011. APOBEC2, a selective inhibitor of TGF $\beta$ signaling, regulates left-right axis specification during early embryogenesis. Dev. Biol. 350, 13-23. https://doi.org/10.1016/j.ydbio.2010.09.016

10. Mikl M.C., Watt I.N., Lu M., Reik W., Davies S.L., Neuberger M.S., Rada C. 2005. Mice deficient in APOBEC2 and APOBEC3. Mol. Cell. Biol. 25, 72707277.

11. Sato Y., Ohtsubo H., Nihei N., Kaneko T., Sato Y., Adachi S.I., Kondo S., Nakamura M., Mizunoya W., Iida H., Tatsumi R., Rada C., Yoshizawa F. 2018. Apobec 2 deficiency causes mitochondrial defects and mitophagy in skeletal muscle. FASEB J. 32 (3), 14281439. https://doi.org/10.1096/fj.201700493R

12. Rogozin I., Basu M., Jordan I., Pavlov Y., Koonin E. 2005. APOBEC4, a new member of the AID/APOBEC family of polynucleotide (deoxy)cytidine deaminases predicted by computational analysis. Cell Cycle. 4, 1281-1285.

https://doi.org/10.4161/cc.4.9.1994

13. Pilzecker B., Jacobs H. 2019. Mutating for good: DNA damage responses during somatic hypermutation. Front. Immunol. 10, 438. https://doi.org/10.3389/fimmu.2019.00438

14. Sharbeen G., Yee C., Smith A., Jolly C. 2012. Ectopic restriction of DNA repair reveals that UNG2 excites AID-induced uracils predominantly or exclusively during G1 phase. J. Exp. Med. 209, 965-974. https://doi.org/10.1084/jem20112379

15. Wang Q., Kieffer-Kwon K., Oliviera T., Mayer C., Yao K., Pai J., Cao Z., Dose M., Casellas R., Jankovic M., Nussenzweig M.C., Robbiani D.F. 2017. The cell cycle restricts activation-induced cytidine deaminase activity to early G1. J. Exp. Med. 214, 49-58. https://doi.org/10.1084/jem20161649

16. Yu K., Lieber M. 2019. Current insights into the mechanism of mammalian immunoglobulin class switch recombination. Crit. Rev. Biochem. Mol. Biol. 54(4), 333-351. https://doi.org/10.1080/10409238.2019.1659227

17. Zan H., Casali P. 2013. Regulation of Aicda expression and AID activity. Autoimmunity. 46 (2), 83-101. https://doi.org/10.3109/08916934.2012.749244

18. Lackey L., Law E., Brown W., Harris R. 2013. Subcellular localization of the APOBEC3 proteins during mitosis and implications for genomic DNA deamination. Cell Cycle. 12 (5), 762-772. https://doi.org/10.4161/cc. 23713

19. Patenaude A., Orthwein A., Hu Y., Campo V., Kavli B., Buschiazzo A., Di Noia J. 2009. Active nuclear import and cytoplasmic retention of activation-induced deaminase. Nat. Struct. Mol. Biol. 16 (5), 517-527. https://doi.org/10.1038/nsmb.1598

20. Patenaude A., Di Noia J. 2010. The mechanisms regulating the subcellular localization of AID. Nucleus. 1 (4), 325-331. https://doi.org/10.4161/nucl.1.4.12107

21. Le Q., Maizels N. 2019. Activation-induced deaminase (AID) localizes to the nucleus in brief pulses. PLoS Genet. 15 (2), e1007968. https://doi.org/10.1371/journal.pgen.1007968

22. Pasqualucci L., Kitaura Y., Gu H., Dalla-Favera R. 2006. PKA-mediated phosphorylation regulates the function of activation-induced deaminase (AID) in B cells. Proc. Natl. Acad. Sci. U. S. A. 103 (2), 395-400. https://doi.org/10.1073/pnas.0509969103

23. Basu U., Chaudhuri J., Alpert C., Dutt S., Ranganath S., Li G., Schrum J., Manis J., Alt F. 2005. The AID antibody diversification enzyme is regulated by protein kinase A phosphorylation. Nature. 438 (7067), 508-511. https://doi.org/10.1038/nature04255

24. Cheng H., Vuong B., Basu U., Franklin A., Schwer B., Astarita J., Phan R.T., Datta A., Manis J., Alt F.W., Chaudhuri J. 2009. Integrity of the AID serine-38 phosphorylation site is critical for class switch recombination and somatic hypermutation in mice. Proc. Natl. Acad. Sci. U. S. A. 106 (8), 2717-2722. https://doi.org/10.1073/pnas.0812304106 
25. Blanc V., Henderson J., Kennedy S, Davidson N. 2001. Mutagenesis of apobec-1 complementation factor reveals distinct domains that modulate RNA binding, protein-protein interaction with apobec-1, and complementation of $\mathrm{C}$ to $\mathrm{U}$ RNA-editing activity. J. Biol. Chem. 276 (49), 46386-46393. https://doi.org/10.1074/jbc.M107654200

26. Blanc V., Henderson J., Kennedy S, Davidson N. 2003. A novel nuclear localization signal in the auxiliary domain of apobec- 1 complementation factor regulates nucleocytoplasmic import and shuttling. J. Biol. Chem. 278 (42), 41198-41204. https://doi.org/10.1074/jbc.M302951200

27. Nowarski R., Wilner O.I., Cheshin O., Shahar O.D., Kenig E., Baraz L., Britan-Rosich E., Nagler A., Harris R.S., Goldberg M., Willner I., Kotler M. 2012. APOBEC3G enhances lymphoma cell radioresistance by promoting cytidine deaminase-dependent DNA repair. Blood. 120, 366-375.

https://doi.org/10.1182/blood-2012-01-402123

28. Brar S.S., Watson M., Diaz M. 2004. Activation-induced cytosine deaminase (AID) is actively exported out of the nucleus but retained by the induction of DNA breaks. J. Biol. Chem. 279, 26395-26401. https://doi.org/10.1074/jbc.M403503200

29. Wakae K., Kondo S., Pham H.T., Wakisaka N., Que L., Li Y., Zheng X., Fukano K., Kitamura K., Watashi K., Aizaki H., Ueno T., Moriyama-Kita M., Ishikawa K., Nakanishi Y., et al. 2020. EBV-LMP1 induces APOBEC3s and mitochondrial DNA hypermutation in nasopharyngeal cancer. Cancer Med. 9 (20), 76637671. https://doi.org/10.1002/cam4.3357

30. Wu H., Zhang K., Chen Y., Li J., Strout M.P., Gu X. 2020. Optimized high-fidelity 3DPCR to assess potential mitochondrial targeting by activation-induced cytidine deaminase. FEBS Open Bio. 10 (9), 17821792.

https://doi.org/10.1002/2211-5463.12927

31. Suspène R., Aynaud M.M., Guétard D., Henry M., Eckhoff G., Marchio A., Pineau P., Dejean A., Vartanian J.P., Wain-Hobson S. 2011. Somatic hypermutation of human mitochondrial and nuclear DNA by APOBEC3 cytidine deaminases, a pathway for DNA catabolism. Proc. Natl. Acad. Sci. U. S. A. 108(12), 4858-4863. https://doi.org/10.1073/pnas.1009687108

32. Grundström C., Kumar A., Priya A., Negi N., Grundström T. 2018. S1 and PAX5 transcription factors recruit AID to Igh DNA. Eur. J. Immunol. 48 (10), 1687-1697. https://doi.org/10.1002/eji.201847625

33. Garrett-Sinha L.A. 2013. Review of Ets1 structure, function, and roles in immunity. Cell. Mol. Life Sci. 70 (18), 3375-3390. https://doi.org/10.1007/s00018-012-1243-7

34. John S.A., Clements J.L., Russell L.M., GarrettSinha L.A. 2007. Ets-1 regulates plasma cell differentiation by interfering with the activity of the transcription factor Blimp-1. J. Biol. Chem. 283 (2), 951-962. https://doi.org/10.1074/jbc.M705262200

35. Shaffer A.L., Lin K.I., Kuo T.C., Yu X., Hurt E.M., Rosenwald A., Giltnane J.M., Yang L., Zhao H., Cal- ame K., Staudt L.M. 2002. Blimp-1 orchestrates plasma cell differentiation by extinguishing the mature B cell gene expression program. Immunity. 17 (1), 51-62. https://doi.org/10.1016/s1074-7613(02)00335-7

36. Meng F.L., Du Z., Federation A., Hu J., Wang Q., Kieffer-Kwon K.R., Meyers R.M., Amor C., Wasserman C.R., Neuberg D., Casellas R., Nussenzweig M.C., Bradner J.E., Liu X.S., Alt F.W. 2014. Convergent transcription at intragenic super-enhancers targets AID-initiated genomic instability. Cell. 159 (7), 1538-1548. https://doi.org/10.1016/j.cell.2014.11.014

37. Qian J., Wang Q., Dose M., Pruett N., KiefferKwon K.R., Resch W., Liang G., Tang Z., Mathé E., Benner C., Dubois W., Nelson S., Vian L., Oliveira T.Y., Jankovic M., et al. 2014. B cell super-enhancers and regulatory clusters recruit AID tumorigenic activity. Cell. 159 (7), 1524-1537. https://doi.org/10.1016/j.cell.2014.11.013

38. Mangeat B., Turelli P., Caron G., Friedli M., Perrin L., Trono D. 2003. Broad antiretroviral defence by human APOBEC3G through lethal editing of nascent reverse transcripts. Nature. 424 (6944), 99-103.

39. Yang B., Chen K., Zhang C., Huang S., Zhang H. 2007. Virion-associated uracil DNA glycosylase-2 and apurinic/apyrimidinic endonuclease are involved in the degradation of APOBEC3G-edited nascent HIV-1 DNA. J. Biol. Chem. 282 (16), 11667-11675. https://doi.org/10.1074/jbc.M606864200

40. Bishop K., Verma M., Kim E., Wolinsky S., Malim M. 2008. APOBEC3G inhibits elongation of HIV-1 reverse transcripts. PLoS Pathog. 4 (12), e1000231. https://doi.org/10.1371/journal.ppat.1000231

41. Browne E.P., Allers C., Landau N.R. 2009. Restriction of HIV-1 by APOBEC $3 \mathrm{G}$ is cytidine deaminasedependent. Virology. 387 (2), 313-321. doi . 02.026 https://doi.org/10.1016/j.virol.2009

42. Albin J.S., Brown W.L., Harris R.S. 2014. Catalytic activity of APOBEC3F is required for efficient restriction of Vif-deficient human immunodeficiency virus. Virology. 450-451, 49-54. https://doi.org/10.1016/j.virol.2013.11.041

43. Derse D., Hill S.A., Princler G., Lloyd P., Heidecker G. 2007. Resistance of human T cell leukemia virus type 1 to APOBEC3G restriction is mediated by elements in nucleocapsid. Proc. Natl. Acad. Sci. U. S. A. 104 (8), 2915-2920. https://doi.org/10.1073/pnas.0609444104

44. Russell R.A., Wiegand H.L., Moore M.D., Schäfer A., McClure M.O., Cullen B.R. 2005. Foamy virus Bet proteins function as novel inhibitors of the APOBEC3 family of innate antiretroviral defense factors. J. Virol. 79 (14), 8724-8731. . https://doi.org/10.1128/JVI.79.14.8724-8731.2005

45. Desimmie B.A., Delviks-Frankenberrry K.A., Burdick R.C., Qi D., Izumi T., Pathak V.K. 2014. Multiple APOBEC3 restriction factors for HIV-1 and one Vif to rule them all. J. Mol. Biol. 426 (6), 1220-1245. https://doi.org/10.1016/j.jmb.2013.10.033

46. Huthoff H., Malim M.H. 2007. Identification of amino acid residues in APOBEC3G required for regulation by human immunodeficiency virus type 1 Vif and 
virion encapsidation. J. Virol. 81 (8), 3807-3815. https://doi.org/10.1128/JVI.02795-06

47. Letko M., Silvestri G., Hahn B.H., Bibollet-Ruche F., Gokcumen O., Simon V., Ooms M. 2013. Vif proteins from diverse primate lentiviral lineages use the same binding site in APOBEC3G. J. Virol. 87 (21), $11861-11871$. https://doi.org/10.1128/JVI.01944-13

48. Smith J.L., Bu W., Burdick R.C., Pathak V.K. 2009. Multiple ways of targeting APOBEC3-virion infectivity factor interactions for anti-HIV-1 drug development. Trends Pharmacol. Sci. 30 (12), 638-646. https://doi.org/10.1016/j.tips.2009.09.006

49. Ao Z., Wang X., Bello A., Jayappa K.D., Yu Z., Fowke K., He X., Chen X., Li J., Kobinger G., Yao X. 2011. Characterization of anti-HIV activity mediated by R88-APOBEC3G mutant fusion proteins in $\mathrm{CD}^{+} \mathrm{T}$ cells, peripheral blood mononuclear cells, and macrophages. Hum. Gene Ther. 22 (10), 1225-1237. https://doi.org/10.1089/hum.2010.012

50. Delviks-Frankenberry K.A., Ackerman D., Timberlake N.D., Hamscher M., Nikolaitchik O.A., Hu W.S., Torbett B.E., Pathak V.K. 2019. Development of lentiviral vectors for HIV-1 gene therapy with Vif-resistant APOBEC3G. Mol. Ther. Nucleic Acids. 18, 1023-1038. https://doi.org/10.1016/j.omtn.2019.10.024

51. Fourati S., Malet I., Binka M., Boukobza S., Wirden M., Sayon S., Simon A., Katlama C., Simon V., Calvez V., Marcelin A.G. 2010. Partially active HIV-1 Vif alleles facilitate viral escape from specific antiretrovirals. AIDS. 24 (15), 2313-2231. https://doi.org/10.1097/QAD.0b013e32833e515a

52. Kim E.Y., Lorenzo-Redondo R., Little S.J., Chung Y.S., Phalora P.K., Maljkovic Berry I., Archer J., Penugonda S., Fischer W., Richman D.D., Bhattacharya T., Malim M.H., Wolinsky S.M. 2014. Human APOBEC3 induced mutation of human immunodeficiency virus type- 1 contributes to adaptation and evolution in natural infection. PLoS Pathog. 10 (7), e 1004281. https://doi.org/10.1371/journal.ppat.1004281

53. Chen H., Lilley C., Yu Q., Lee D., Chou J., Narvaiza I., Landau N.R., Weitzman M.D. 2006. APOBEC3A is a potent inhibitor of adeno-associated virus and retrotransposons. Curr. Biol. 16 (5), 480-485. https://doi.org/10.1016/j.cub.2006.01.031

54. Yu Q., Chen D., König R., Mariani R., Unutmaz D., Landau N.R. 2004. APOBEC3B and APOBEC3C are potent inhibitors of simian immunodeficiency virus replication. J. Biol. Chem. 279 (51), 53379-53386. https://doi.org/10.1074/jbc.M408802200

55. Zielonka J., Bravo I.G., Marino D., Conrad E., Perković M., Battenberg M., Cichutek K., Münk C. 2009. Restriction of equine infectious anemia virus by equine APOBEC3 cytidine deaminases. J. Virol. 83, 7547-7559. https://doi.org/10.1128/JVI.00015-09

56. Bishop K., Holmes R., Sheehy A., Davidson N., Cho S., Malim M.H. 2004. Cytidine deamination of retroviral DNA by diverse APOBEC proteins. Curr. Biol. 14 (15), 1392-1396.

https://doi.org/10.1016/j.cub.2004.06.057
57. Löchelt M., Romen F., Bastone P., Muckenfuss H., Kirchner N., Kim Y., Truyen U., Rösler U., Battenberg M., Saib A., Flory E., Cichutek K., Münk C. 2005. The antiretroviral activity of APOBEC3 is inhibited by the foamy virus accessory Bet protein. Proc. Natl. Acad. Sci. U. S. A. 102 (22), 7982-7987. https://doi.org/10.1073/pnas.0501445102

58. Delebecque F., Suspène R., Calattini S., Casartelli N., Saïb A., Froment A., Wain-Hobson S., Gessain A., Vartanian J.P., Schwartz O. 2006. Restriction of foamy viruses by APOBEC cytidine deaminases. $J$. $\mathrm{Vi}$ rol. 80 (2), 605-614. https://doi.org/10.1128/JVI.80.2.605-614.2006

59. Noguchi C., Ishino H., Tsuge M., Fujimoto Y., Imamura M., Takahashi S., Chayama K. 2005. G to A hypermutation of hepatitis B virus. Hepatology. 41 (3), $626-633$. https://doi.org/10.1002/hep.20580

60. Suspène R., Guétard D., Henry M., Sommer P., Wain-Hobson S., Vartanian J.P. 2005. Extensive editing of both hepatitis B virus DNA strands by APOBEC3 cytidine deaminases in vitro and in vivo. Proc. Natl. Acad. Sci. U. S. A. 102, 8321-8326. https://doi.org/10.1073/pnas.0408223102

61. Bishop K., Holmes R., Sheehy A.M., Malim M.H. 2004. APOBEC-mediated editing of viral RNA. Science. 305 (5684), 645. https://doi.org/10.1126/science. 1100658

62. Sharma S., Patnaik S., Taggart T., Kannisto E., Enriquez S., Gollnick P., Baysal B. 2015. APOBEC3A cytidine deaminase induces RNA editing in monocytes and macrophages. Nat. Commun. 6, 6881. https://doi.org/10.1038/ncomms7881

63. Asaoka M., Ishikawa T., Takabe K., Patnaik S.K. 2019. APOBEC3-mediated RNA editing in breast cancer is associated with heightened immune activity and improved survival. Int. J. Mol. Sci. 20 (22), 5621. https://doi.org/10.3390/ijms20225621

64. Caval V., Jiao W., Berry N., Khalfi P., Pitré E., Thiers V., Vartanian J.P., Wain-Hobson S., Suspène R. 2019. Mouse APOBEC1 cytidine deaminase can induce somatic mutations in chromosomal DNA. BMC Genomics. 20 (1), 858. https://doi.org/10.1186/s12864-019-6216-X

65. Fehrholz M., Kendl S., Prifert C., Weissbrich B., Lemon K., Rennick L., Duprex P.W., Rima B.K., Koning F.A., Holmes R.K., Malim M.H., SchneiderSchaulies J. 2012. The innate antiviral factor APOBEC3G targets replication of measles, mumps and respiratory syncytial viruses. J. Gen. Virol. 93 (3), $565-576$. https://doi.org/10.1099/vir.0.038919-0

66. Milewska A., Kindler E., Vkovski P., Zeglen S., Ochman M., Thiel V., Rajfur Z., Pyr K. 2018. APOBEC3mediated restriction of RNA virus replication. Sci. Rep. 8, 5960. https://doi.org/10.1038/s41598-018-24448-2

67. Burns M.B., Temiz N.A., Harris R.S. 2013. Evidence for APOBEC3B mutagenesis in multiple human cancers. Nat. Genet. 45 (9), 977-983. https://doi.org/10.1038/ng.2701

68. Campbell P.J., Getz G., Korbel J.O., ICGC/TCGA Pan-Cancer Analysis of Whole Genomes Consortium. 
2020. Pan-cancer analysis of whole genomes. Nature. 578, 82-93.

https://doi.org/10.1038/s41586-020-1969-6

69. Went M., Kinnersley B., Sud A., Johnson D.C., Weinhold N., Försti A., van Duin M., Orlando G., Mitchell J.S., Kuiper R., Walker B.A., Gregory W.M., Hoffmann P., Jackson G.H., Nöthen M.M., et al. 2019. Transcriptome-wide association study of multiple myeloma identifies candidate susceptibility genes. Hum. Genomics. 13 (1), 37. https://doi.org/10.1186/s40246-019-0231-5

70. Nik-Zainal S., Wedge D.C., Alexandrov L.B., Petljak M., Butler A.P., Bolli N., Davies H.R., Knappskog S., Martin S., Papaemmanuil E., Ramakrishna M., Shlien A., Simonic I., Xue Y., Tyler-Smith C., et al. 2014. Association of a germline copy number polymorphism of APOBEC 3A and APOBEC3B with burden of putative APOBEC-dependent mutations in breast cancer. Nat. Genet. 46 (5), 487-491. https://doi.org/10.1038/ng.2955

71. Middlebrooks C., Banday A., Matsuda K., Udquim K.I., Onabajo O.O., Paquin A., Figueroa J.D., Zhu B., Koutros S., Kubo M., Shuin T., Freedman N.D., Kogevinas M., Malats N., Chanock S.J., et al. 2016. Association of germline variants in the APOBEC 3 region with cancer risk and enrichment with APOBEC-signature mutations in tumors. Nat. Genet. 48 (11), $1330-1338$. https://doi.org/10.1038/ng.3670

72. Cortez L.M., Brown A.L., Dennis M.A., Collins C.D., Brown A.J., Mitchell D., Mertz T.M., Roberts S.A. 2019. APOBEC3A is a prominent cytidine deaminase in breast cancer. PLoS Genet. 15 (12), e 1008545. https://doi.org/10.1371/journal.pgen.1008545

73. Yoshikawa K., Okazaki I.M., Eto T., Kinoshita K., Muramatsu M., Nagaoka H., Honjo T. 2002. AID enzyme-induced hypermutation in an actively transcribed gene in fibroblasts. Science. 296, 2033-2036. https://doi.org/10.1126/science.1071556

74. Kotani A., Okazaki I., Muramatsu M., Kinoshita K., Begum N.A., Nakajima T., Saito H., Honjo T. 2005. A target selection of somatic hypermutations is regulated similarly between $\mathrm{T}$ and $\mathrm{B}$ cells upon activation-induced cytidine deaminase expression. Proc. Natl. Acad. Sci. U. S. A. 102, 4506-4511.

https://doi.org/10.1073/pnas.0500830102

75. Okazaki I., Hiai H., Kakazu N., Yamada S., Muramatsu M., Kinoshita K., Honjo T. 2003. Constitutive expression of AID leads to tumorigenesis. J. Exp. Med. 197, 1173-1181. https://doi.org/10.1084/jem.20030275

76. McCarthy H., Wierda W., Barron L., Cromwell C.C., Wang J., Coombes K.R., Rangel R., Elenitoba-Johnson K.S., Keating M.J., Abruzzo L.V. 2003. High expression of activation-induced cytidine deaminase (AID. and splice variants is a distinctive feature of poor-prognosis chronic lymphocytic leukemia. Blood. 101, 4903-4908.

https://doi.org/10.1182/blood-2002-09-2906

77. Heintel D., Kroemer E., Kienle D., Schwarzinger I., Gleiss A., Schwarzmeier J., Marculescu R., Le T., Mannhalter C., Gaiger A., Stilgenbauer S., Döhner H., Fonatsch C., Jäger U.; the German CLL Study
Group. 2004. High expression of activation-induced cytidine deaminase (AID) mRNA is associated with unmutated IGVH gene status and unfavourable cytogenetic aberrations in patients with chronic lymphocytic leukaemia. Leukemia. 18, 756-762.

https://doi.org/10.1038/sj.leu.2403294

78. Wu X., Darce J., Chang S., Nowakowski G., Jelinek D. 2008. Alternative splicing regulates activation-induced cytidine deaminase (AID): implications for suppression of AID mutagenic activity in normal and malignant B cells. Blood. 112 (12), 4675-4682.

https://doi.org/10.1182/blood-2008-03-145995

79. Marantidou F., Dagklis A., Stalika E., Korkolopoulou P., Saetta A., Anagnostopoulos A., Laoutaris N., Stamatopoulos K., Belessi C., Scouras Z., Patsouris E. 2010. Activation-induced cytidine deaminase splicing patterns in chronic lymphocytic leukemia. Blood Cells Mol. Dis. 44 (4), 262-267.

https://doi.org/10.1016/j.bcmd.2009.12.005

80. Rebhandl S., Huemer M., Greil R., Geisberger R. 2015. AID/APOBEC deaminases and cancer. Oncoscience. 2 (4), 320-333. doi https://doi.org/10.18632/oncoscience

81. Matsumoto Y., Marusawa H., Kinoshita K., Endo Y., Kou T., Morisawa T., Azuma T., Okazaki I.M., Honjo T., Chiba T. 2007. Helicobacter pylori infection triggers aberrant expression of activation-induced cytidine deaminase in gastric epithelium. Nat. Med. 13 (4), 470-476. https://doi.org/10.1038/nm1566

82. Maruyama W., Shirakawa K., Matsui H., Matsumoto T., Yamazaki H., Sarca A.D., Kazuma Y., Kobayashi M., Shindo K., Takaori-Kondo A. 2016. Classical NF-кB pathway is responsible for APOBEC 3B expression in cancer cells. Biochem. Biophys. Res. Commun. 478 (3), $1466-1471$.

https://doi.org/10.1016/j.bbrc.2016.08.148

83. Gao J., Choudhry H., Cao W. 2018. Apolipoprotein B mRNA editing enzyme catalytic polypeptide-like family genes activation and regulation during tumorigenesis. Cancer Sci. 109 (8), 2375-2382. https://doi.org/10.1111/cas.13658

84. Maul R.W., Gearhart P.J. 2010. Aid and somatic hypermutation. Adv. Immunol. 105, 159-191. https://doi.org/10.1016/S0065-2776(10)05006-6

85. Tilborghs S., Corthouts J., Verhoeven Y., Arias D., Rolfo C., Trinh X., van Dam P. 2017. The role of Nuclear Factor-kappa B signaling in human cervical cancer. Crit. Rev. Oncol. Hematol. 120, 141-150. https://doi.org/10.1016/j.critrevonc.2017.11.001

86. Tanaka M., Marusawa H., Seno H., Matsumoto Y., Ueda Y., Kodama Y., Endo Y., Yamauchi J., Matsumoto T., Takaori-Kondo A., Ikai I., Chiba T. 2006. Anti-viral protein APOBEC3G is induced by interferon-alpha stimulation in human hepatocytes. Biochem. Biophys. Res. Commun. 341 (2), 314-319. https://doi.org/10.1016/j.bbrc.2005.12.192

87. Pillai S., Abdel-Mohsen M., Guatelli J., Skasko M., Monto A., Fujimoto K., Yukl S., Greene W.C., Kovari H., Rauch A., Fellay J., Battegay M., Hirschel B., Witteck A., Bernasconi E., et al. 2012. Role of retroviral restriction factors in the interferon- $\alpha$-mediated suppression of HIV-1 in vivo. Proc. Natl. Acad. Sci. 
U. S. A. 109 (8), 3035-3040. https://doi.org/10.1073/pnas.1111573109

88. Li Y., Xia Y., Han M., Chen G., Zhang D., Thasler W., Protzer U., Ning Q. 2017. IFN- $\alpha$-mediated base excision repair pathway correlates with antiviral response against hepatitis B virus infection. Sci. Rep. 7, 12715. https://doi.org/10.1038/s41598-017-13082-z

89. Bobrovnitchaia I., Valieris R., Drummond R., Lima J., Freitas H., Bartelli T., de Amorim M., Nunes D., Dias-Neto E., Silva I. 2020. APOBEC-mediated DNA alterations: a possible new mechanism of carcinogenesis in EBV-positive gastric cancer. Int. J. Cancer. 146 (1), 181-191. https://doi.org/10.1002/ijc.32411

90. He B., Raab-Traub N., Casali P., Cerutti A. 2003. EBV-encoded latent membrane protein 1 cooperates with BAFF/BLyS and APRIL to induce T cell-independent Ig heavy chain class switching. J. Immunol. 171, 5215-5224.

https://doi.org/10.4049/jimmunol.171.10.5215

91. Li M., Maizels N. 1999. Activation and targeting of immunoglobulin switch recombination by activities induced by EBV infection. J. Immunol. 163, 6659-6664.

92. Machida K., Cheng K., Sung V., Shimodaira S., Lindsay L., Levine A., Lai M. 2004. Hepatitis C virus induces a mutator phenotype: enhanced mutations of immunoglobulin and protooncogenes. Proc. Natl. Acad. Sci. U. S. A. 101, 4262-4267. https://doi.org/10.1073/pnas.0303971101

93. Kinoshita K., Nonaka T. 2006. The dark side of activation-induced cytidine deaminase: Relationship with leukemia and beyond. Int. J. Hematol. 83 (3), 201-207. https://doi.org/10.1532/IJH97.06011

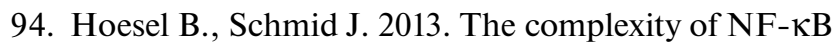
signaling in inflammation and cancer. Mol. Cancer. $12,86$.

https://doi.org/10.1186/1476-4598-12-86

95. Siriwardena S., Chen K., Bhagwat A. 2016. The functions and malfunctions of AID/APOBEC family deaminases: The known knowns and the known unknowns. Chem. Rev. 116 (20), 12688-12710. https://doi.org/10.1021/acs.chemrev.6b00296

96. Wang Y., Wang X., Zhang H., Zhou L., Liu S., Kolson D., Song L., Ye L., Ho W. 2009. Expression and regulation of antiviral protein APOBEC3G in human neuronal cells. J. Neuroimmunol. 206 (1-2), 14-21. https://doi.org/10.1016/j.jneuroim.2008.10.003

97. Endo Y., Marusawa H., Kou T., Nakase H., Fujii S., Fujimori T., Kinoshita K., Honjo T., Chiba T. 2008. Activation-induced cytidine deaminase links between inflammation and the development of colitis-associated colorectal cancers. Gastroenterology. 135 (3), 889-898, 898.e1-3.

https://doi.org/10.1053/j.gastro.2008.06.091

98. Marusawa H., Chiba T. 2010. Helicobacter pylori-induced activation-induced cytidine deaminase expression and carcinogenesis. Curr. Opin. Immunol. 22 (4), 442-447.

https://doi.org/10.1016/j.coi.2010.06.001
99. Sugiyama T., Asaka M. 2004. Helicobacter pylori infection and gastric cancer. Med. Electron. Microsc. 37 (3), 149-157. https://doi.org/10.1007/s00795-004-0250-7

100. Eaden J., Abrams K., Mayberry J. 2001. The risk of colorectal cancer in ulcerative colitis: a meta-analysis. Gut. 48, 526-535. https://doi.org/10.1136/gut.48.4.526

101. Jess T., Gamborg M., Matzen P., Munkholm P., Sorensen T. 2005. Increased risk of intestinal cancer in Crohn's disease: a metaanalysis of population-based cohort studies. Am. J. Gastroenterol. 100, 2724-2729. https://doi.org/10.1111/j.1572-0241.2005.00287.x

102. Zou J., Wang C., Ma X., Wang E., Peng G. 2017. APOBEC3B, a molecular driver of mutagenesis in human cancers. Cell Biosci. 7, 29. https://doi.org/10.1186/s13578-017-0156-4

103. Periyasamy M., Singh A., Gemma C., Kranjec C., Farzan R. Leach D.A., Navaratnam N., Pálinkás H.L., Vértessy B.G., Fenton T.R., Doorbar J., Fuller-Pace F., Meek D.W., Coombes R.C., Buluwela L., Ali S. 2017. p53 controls expression of the DNA deaminase APOBEC3B to limit its potential mutagenic activity in cancer cells. Nucleic Acids Res. 45 (19), 11056-11069. https://doi.org/10.1093/nar/gkx721

104. Kanu N., Cerone M.A., Goh G., Zalmas L.P., Bartkova J., Dietzen M., McGranahan N., Rogers R., Law E.K., Gromova I., Kschischo M., Walton M.I., Rossanese O.W., Bartek J., Harris R.S., et al. 2016. DNA replication stress mediates APOBEC3 family mutagenesis in breast cancer. Genome Biol. 17 (1), 185. https://doi.org/10.1186/s13059-016-1042-9

105. Roper N., Gao S., Maity T., Banday R., Zhang X., Venugopalan A., Cultraro C.M., Patidar R., Sindiri S., Brown A.L., Goncearenco A., Panchenko A.R., Biswas R., Thomas A., Rajan A., et al. 2019. APOBEC mutagenesis and copy-number alterations are drivers of proteogenomic tumor evolution and heterogeneity in metastatic thoracic tumors. Cell Reports. 26 (10), 2651-2666.e6. https://doi.org/10.1016/j.celrep.2019.02.028

106. Covino D.A., Gauzzi M.C., Fantuzzi L. 2018. Understanding the regulation of APOBEC 3 expression: current evidence and much to learn. J. Leukoc. Biol. 103 (3), 433-444. https://doi.org/10.1002/JLB.2MR0717-310R

107. Warren C., Westrich J., Doorslaer K., Pyeon D. 2017. Roles of APOBEC3A and APOBEC3B in human papillomavirus infection and disease progression. $\mathrm{Vi}$ ruses. 9 (8), 233. https://doi.org/10.3390/v9080233

108. Niavarani A., Shahrabi Farahani A., Sharafkhah M., Rassoulzadegan M. 2018. Pancancer analysis identifies prognostic high-APOBEC1 expression level implicated in cancer in-frame insertions and deletions. Carcinogenesis. 39 (3), 327-335. https://doi.org/10.1093/carcin/bgy005

109. Li A., Wu J., Zhai A., Qian J., Wang X., Qaria M.A., Zhang Q., Li Y., Fang Y., Kao W., Song W., Zhang Z., Zhang F. 2019. HBV triggers APOBEC2 expression through miR-122 regulation and affects the proliferation of liver cancer cells. Int. J. Oncol. 55 (5), 
1137-1148. https://doi.org/10.3892/ijo.2019.4870

110. Cao W., Wu W. 2018. Apolipoprotein B mRNA editing enzyme, catalytic polypeptide-like gene expression, RNA editing, and microRNAs regulation. Methods Mol. Biol. 1699, 75-81.

https://doi.org/10.1007/978-1-4939-7435-1_5

111. Boichard A. Pham T., Yeerna H., Goodman A., Tamayo P., Lippman S., Frampton G.M., Tsigelny I.F., Kurzrock R. 2019. APOBEC-related mutagenesis and neo-peptide hydrophobicity: implications for response to immunotherapy. Oncoimmunology. 8 (3), 1550341 . https://doi.org/10.1080/2162402X.2018.1550341

112. Driscoll C.B., Schuelke M.R., Kottke T. 2020. APOBEC3B-mediated corruption of the tumor cell immunopeptidome induces heteroclitic neoepitopes for cancer immunotherapy. Nat. Commun. 11 (1), 790. https://doi.org/10.1038/s41467-020-14568-7

113. Maura F., Petljak M., Lionetti M., Cifola I., Liang W., Pinatel E., Alexandrov L.B., Fullam A., Martincorena I., Dawson K.J., Angelopoulos N., Samur M.K., Szalat R., Zamora J., Tarpey P., et al. 2018. Biological and prognostic impact of APOBEC-induced mutations in the spectrum of plasma cell dyscrasias and multiple myeloma cell lines. Leukemia. 32 (4), 1044-1048. https://doi.org/10.1038/leu.2017.345

114. Walker B.A., Wardell C.P., Murison A., Boyle E.M., Begum D.B., Dahir N.M., Proszek P.Z., Melchor L., Pawlyn C., Kaiser M.F., Johnson D.C., Qiang Y.W., Jones J.R., Cairns D.A., Gregory W.M., et al. 2015. APOBEC family mutational signatures are associated with poor prognosis translocations in multiple myeloma. Nat. Commun. 6, 6997.

115. Gara S.K., Tyagi M.V., Patel D.T., Gaskins K., Lack J., Liu Y., Kebebew E. 2020. GATA3 and APOBEC3B are prognostic markers in adrenocortical carcinoma and APOBEC $3 \mathrm{~B}$ is directly transcriptionally regulated by GATA3. Oncotarget. 11, 3354-3370.

https://doi.org/10.18632/oncotarget.27703
116. Du Y., Tao X., Wu J., Yu H., Yu Y., Zhao H. 2018. APOBEC3B up-regulation independently predicts ovarian cancer prognosis: a cohort study. Cancer Cell Int. 18, 78. https://doi.org/10.1186/s12935-018-0572-5

117. Han L., Diao L., Yu S., Xu X., Li J., Zhang R., Yang Y., Werner H.M.J., Eterovic A.K., Yuan Y., Li J., Nair N., Minelli R., Tsang Y.H., Cheung L.W.T, et al. 2015. The genomic landscape and clinical relevance of A-to-I RNA editing in human cancers. Cancer Cell. 28 (4), 515-528. https://doi.org/10.1016/j.ccell.2015.08.013

118. Galeano F., Tomaselli S., Locatelli F., Gallo A. 2012. A-to-I RNA editing: the "ADAR" side of human cancer. Semin. Cell Dev. Biol. 23 (3), 244-250. https://doi.org/10.1016/j.semcdb.2011.09.003

119. Skuse G.R., Cappione A.J., Sowden M., Metheny L.J., Smith H.C. 1996. The neurofibromatosis type I messenger RNA undergoes base-modification RNA editing. Nucleic Acids Res. 24 (3), 478-485. https://doi.org/10.1093/nar/24.3.478

120. Mukhopadhyay D., Anant S., Lee R.M., Kennedy S., Viskochil D., Davidson N.O. 2002. C $\rightarrow \mathrm{U}$ editing of neurofibromatosis $1 \mathrm{mRNA}$ occurs in tumors that express both the type II transcript and apobec-1, the catalytic subunit of the apolipoprotein B mRNA-editing enzyme. Am. J. Hum. Genet. 70 (1), 38-50. https://doi.org/10.1086/337952

121. Sharma S., Patnaik S.K., Kemer Z., Baysal B.E. 2017. Transient overexpression of exogenous APOBEC3A causes C-to-U RNA editing of thousands of genes. RNA Biol. 14 (5), 603-610. https://doi.org/10.1080/15476286.2016.1184387

122. Baysal B.E., Sharma S., Hashemikhabir S., Jang S.C. 2017. RNA editing in pathogenesis of cancer. Cancer Res. 77 (14), 3733-3739. https://doi.org/10.1158/0008-5472.CAN-17-0520

Translated by N. Onishchenko 